Application of the TraPPE force field to predicting isothermal pressure-volume curves at high pressures and high temperatures

B. L. Eggimann, J. I. Siepmann, L. E. Fried

May 23, 2006

International Journal of Thermophysics 
This document was prepared as an account of work sponsored by an agency of the United States Government. Neither the United States Government nor the University of California nor any of their employees, makes any warranty, express or implied, or assumes any legal liability or responsibility for the accuracy, completeness, or usefulness of any information, apparatus, product, or process disclosed, or represents that its use would not infringe privately owned rights. Reference herein to any specific commercial product, process, or service by trade name, trademark, manufacturer, or otherwise, does not necessarily constitute or imply its endorsement, recommendation, or favoring by the United States Government or the University of California. The views and opinions of authors expressed herein do not necessarily state or reflect those of the United States Government or the University of California, and shall not be used for advertising or product endorsement purposes. 


\title{
Application of the TraPPE force field to predicting isothermal pressure-volume curves at high pressures and high temperatures ${ }^{1}$
}

\author{
Becky L. Eggimann ${ }^{2}$, J. Ilja Siepmann ${ }^{2,3}$, and L.E. Fried ${ }^{3,4}$
}

${ }^{1}$ Paper presented at the Sixteenth Symposium on Thermophysical Properties, July 30August 4, 2006, Boulder, Colorado, U.S.A.

${ }^{2}$ Departments of Chemistry and of Chemical Engineering and Materials Science, University of Minnesota, 207 Pleasant Street SE, Minneapolis, Minnesota 55455-0431 U.S.A

3 To whom correspondence should be addressed. E-mail: siepmann@chem.umn.edu and lfried@llnl.gov

${ }^{4}$ Chemistry and Materials Science Directorate, Lawrence Livermore National Laboratory, Livermore, California 94550 U.S.A. 


\section{ABSTRACT}

Knowledge of the thermophysical properties of materials at extreme pressure and temperature conditions is essential for improving our understanding of many planetary and detonation processes. Significant gaps in what is known about the behavior of materials at high density and high temperature exist, largely due to the limitations and dangers of performing experiments at the necessary extreme conditions. Modelling these systems through the use of equations of state and particle-based simulation methods significantly extends the range of pressures and temperatures that can be safely studied. The reliability of such calculations depends on the accuracy of the models used. Here we present an assessment of the united-atom version of the TraPPE (Transferable Potentials for Phase Equilibria) force field and single-site exp-6 representations for methane, methanol, oxygen, and ammonia at extreme conditions. As shown by Monte Carlo simulations in the isobaricisothermal ensemble, the TraPPE models, despite being parameterized to the vapor-liquid coexistence curve (i.e. relatively mild conditions), perform remarkably well in the high pressure/high temperature regime. The single-site exp-6 models can fit experimental data in the high pressure/temperature regime very well, but the parameters are less transferable to ambient conditions.

KEY WORDS: equation of state, extreme conditions, Monte Carlo simulation, transferable force field 


\section{INTRODUCTION}

Many of the most common elements and molecules in the universe exist in highly compressed environments. The interior of planets and stars account for far more matter than the exterior surfaces, yet comparatively little is known about the thermophysical properties of small molecules under such extreme pressures and temperatures. At the high densities of planetary interiors, molecular properties can be very different from those observed at the ambient conditions of the Earth's surface [1]. A more complete understanding of the thermophysics of systems in the high pressure and temperature regime has value for a wide range of disciplines, including planetary physics, detonation processes, and materials science $[2]$.

Despite the importance of studying systems at extreme pressures and temperatures, the dangers and challenges of performing experiments under those conditions are well known and not easily overcome. While modelling via equations of state or particle-based simulations can be a convenient alternative, it does not come without its own limitations. The predictions made by using either analytical equations of state or molecular simulations are only as accurate as the underlying models they employ. With poor availability of experimental data, validating these models can be difficult. Most models currently in use, even those used in this study, have been fitted to only a limited set of experimental data. Models that make use of transferable parameters (i.e. the same parameters are fitted to be accurate at several state points, including those beyond the constraints of the initial parameterization conditions) are one option for supplementing the low levels of experimental data available for high pressure/high temperature systems. With transferability as one of its explicit goals, the Transferable Potentials for Phase Equilibria (TraPPE) force field has been developed to reproduce vapor-liquid coexistence curves (i.e. temperatures and pressures well below the extreme region) over a wide range of chemical systems and complexities [3-8]. The TraPPE models have been shown to be reasonably accurate for

several systems beyond the state points and molecules used in the parameter fitting $[9,10]$. 
In the present work, the high-pressure densities and compressibility factors of $\mathrm{CH}_{4}$, $\mathrm{CH}_{3} \mathrm{OH}, \mathrm{O}_{2}$, and $\mathrm{NH}_{3}$ are computed for the united-atom version of the TraPPE force field and compared with data obtained experimentally and with predictions from an analytical equation of state based on $\exp -6$ models.

\section{METHODS}

\subsection{Monte Carlo simulations}

Monte Carlo simulations were performed in the isobaric-isothermal $(N p T)$ ensemble [11] at state points chosen to correspond to those studied by experiment for $\mathrm{CH}_{4}$, $\mathrm{CH}_{3} \mathrm{OH}, \mathrm{O}_{2}$, and $\mathrm{NH}_{3}$. In each simulation, the system consisted of 1000 molecules periodically replicated in a cubic box. Sampling of the resulting phase space was achieved through translations (all systems), rigid-body rotations [12] (all systems other than $\mathrm{CH}_{4}$ which is modeled with a single interaction site and has no rotational degrees of freedom), configurational-bias moves [4,13] for conformational changes of $\mathrm{CH}_{3} \mathrm{OH}$, and volume exchanges with an external pressure bath [11] using scaled center-of-mass coordinates. For every system, the simulations were equilibrated for at least 20,000 Monte Carlo cycles (where one cycle consists of $N=1000$ randomly selected moves), and the production periods consisted of at least $80,000 \mathrm{MC}$ cycles. For each of the systems, five independent simulations were run at every state point. The properties and standard deviations are calcualted by averaging over these independent runs.

Molecular interactions in the TraPPE-UA force field $[3,6,14,15]$ are described by pairwise-additive Lennard-Jones (LJ) and Coulomb potentials for the non-bonded interactions. Spherical potential truncation at $14 \AA$ and analytical tail corrections [16] are applied to the LJ interactions, and an Ewald sum with parameters set to $\kappa \times L=5.6$ and $K_{\max }=5$ is used to compute the electrostatic interactions [16]. Bonded interactions depend on the specific model employed, but generally consist of fixed bond lengths and 
harmonic bending potentials. For this work, united-atom models were used, meaning that all the atoms in a $\mathrm{CH}_{x}$ group were treated as a single pseudo-atom. Using this methodology, methane consists of a single interaction site placed at the carbon atom of the $\mathrm{CH}_{4}$ group. Methanol's $\mathrm{CH}_{3}$ group is also modeled as a pseudo-atom, while oxygen and hydrogen atoms are modeled explicitly. The oxygen and ammonia model includes an additional charge site located at the bond center and near the nitrogen atom, respectively, and both oxygen and ammonia are rigid models. The TraPPE-UA models consist of one, three, three, and five interaction sites for $\mathrm{CH}_{4}, \mathrm{CH}_{3} \mathrm{OH}, \mathrm{O}_{2}$, and $\mathrm{NH}_{3}$, respectively.

Additional simulations in the isobaric-isothermal ensemble were performed for liquid phases at ambient pressure to consider the transferability of the single-site exp-6 and TraPPE models. The only system that is a liquid at standard conditions (298 K and 1 atm) is methanol, and so the $\mathrm{CH}_{3} \mathrm{OH}$ simulations were performed at this temperature and pressure. The other systems were simulated at $1 \mathrm{~atm}$ with temperatures set to yield a liquid state (i.e. just below their normal boiling points).

\subsection{Equation of state predictions using exp-6 models}

A multi-site representation, such as used in the TraPPE-UA force field, allows the user to assemble new molecules from existing building blocks without the need for any parameter fitting and is also essential for an accurate treatment of fluid structure and dynamics. For small molecules, however, molecular shape often plays a minor role in the equation of state and transport properties. In this case effective isotropic single-site interaction models are often convenient. Highly accurate theories of the free energy of the single-site exp-6 potential have been proposed [17-19]. In the current work, we use

a numerical fit to free energies of the exp-6 fluid calculated from Ross's theory [18] and expressed as a polynomial in suitable variables $[19,20]$. The implementation of such theories is many orders of magnitude faster than particle-based simulations, thereby allowing for the 
Table I. Single-site exp-6 parameters.

\begin{tabular}{cccc}
\hline Molecule & $r[\AA]$ & $\epsilon[\mathrm{K}]$ & $\alpha$ \\
\hline $\mathrm{CH}_{4}$ & 4.319 & 142.5 & 12.13 \\
$\mathrm{CH}_{3} \mathrm{OH}$ & 4.114 & 506.8 & 13.00 \\
$\mathrm{O}_{2}$ & 3.865 & 117.7 & 13.50 \\
$\mathrm{NH}_{3}$ & 3.730 & 251.6 & 11.96 \\
\hline
\end{tabular}

rapid evaluation of thermodynamic properties at state points of interest. As the equation of state used here becomes less accurate for sub-critical conditions, the data for the exp- 6 models at ambient conditions were obtained directly from isobaric-isothermal simulations of the liquid phase. Explicit simulations for the exp-6 models agree very well with the equation of state predictions at supercritical conditions.

The parameters for the single-site exp-6 models are listed in Table I. The $\mathrm{CH}_{4}$ model was simultaneously fit to experimental static compression data of Kortbeek et al. [21] as well as shock compression data of Nellis et al. [22]. Using the analytical equation of state in combination with a heat capacity model, a reference single-site exp-6 interaction potential for $\mathrm{CH}_{3} \mathrm{OH}$ was found by fitting to experimental sound speeds [23]. The model for $\mathrm{O}_{2}$ was developed in a manner similar to the procedure used for $\mathrm{CH}_{3} \mathrm{OH}$. Sound speeds for $\mathrm{O}_{2}$ at pressures up to 10 GPa have been reported by Abramson et al. [24].

Larry: need details for the ammonia fitting[25]

\section{RESULTS AND DISCUSSION}

Simulated and calculated densities for the TraPPE-UA force field and the single-site exp-6 models are compared to experimental data in Tables II and III. The corresponding compressibility factors, $Z=p V_{m} / R T$, are plotted in Figures 1 and 2 . 
Table II. Specific densities for $\mathrm{CH}_{4}$ and $\mathrm{NH}_{3}$. Subscripts denote the standard deviation in the last digit for simulation data.

\begin{tabular}{|c|c|c|c|c|c|c|}
\hline \multirow[b]{2}{*}{ Molecule } & \multirow[b]{2}{*}{$T(\mathrm{~K})$} & \multirow[b]{2}{*}{$p(\mathrm{MPa})$} & \multicolumn{2}{|c|}{ Simulation } & \multirow{2}{*}{$\begin{array}{l}\text { Equation of State } \\
\qquad \exp -6 \\
\rho\left(\mathrm{g} \cdot \mathrm{cm}^{-3}\right)\end{array}$} & \multirow{2}{*}{$\begin{array}{l}\text { Experiment } \\
\rho\left(\mathrm{g} \cdot \mathrm{cm}^{-3}\right)\end{array}$} \\
\hline & & & $\begin{array}{c}\text { TraPPE-UA } \\
\rho\left(\mathrm{g} \cdot \mathrm{cm}^{-3}\right)\end{array}$ & $\begin{array}{c}\exp -6 \\
\rho\left(\mathrm{g} \cdot \mathrm{cm}^{-3}\right)\end{array}$ & & \\
\hline \multirow[t]{7}{*}{$\mathrm{CH}_{4}$} & 110 & 0.101 & $0.4232_{5}$ & $0.4006_{6}$ & & $0.4248[26]$ \\
\hline & 298 & 100 & $0.3416_{1}$ & $0.340_{2}$ & 0.336 & $0.341[21]$ \\
\hline & 298 & 400 & $0.4719_{1}$ & $0.468_{2}$ & 0.463 & $0.472[21]$ \\
\hline & 298 & 800 & $0.5433_{1}$ & $0.541_{4}$ & 0.538 & $0.544[21]$ \\
\hline & 298 & 1000 & $0.5677_{1}$ & $0.567_{6}$ & 0.565 & $0.568[21]$ \\
\hline & 298 & 5000 & $0.773_{9}$ & $0.79_{3}$ & & \\
\hline & 298 & 10000 & $0.869_{7}$ & $0.92_{4}$ & & \\
\hline \multirow[t]{8}{*}{$\mathrm{NH}_{3}$} & 223 & 0.101 & $0.699_{2}$ & $0.628_{1}$ & & $0.7023[26]$ \\
\hline & 473 & 100 & $0.4672_{1}$ & $0.454_{4}$ & 0.465 & 0.4928 [25] \\
\hline & 473 & 300 & $0.5941_{5}$ & $0.603_{1}$ & 0.606 & $0.6218[25]$ \\
\hline & 473 & 500 & $0.6550_{1}$ & $0.674_{1}$ & 0.675 & $0.6846[25]$ \\
\hline & 473 & 700 & $0.6970_{2}$ & $0.722_{1}$ & 0.724 & $0.7259[25]$ \\
\hline & 473 & 900 & $0.7298_{1}$ & $0.760_{2}$ & 0.762 & $0.7560[25]$ \\
\hline & 473 & 5000 & $1.017_{1}$ & $1.08_{1}$ & & \\
\hline & 473 & 10000 & $1.140_{3}$ & $1.24_{2}$ & & \\
\hline
\end{tabular}

For $\mathrm{CH}_{4}$, both the TraPPE-UA and the exp-6 models match the experimental data [21] remarkably well at high temperature and pressure, but the exp-6 model shows a somewhat larger deviation for the liquid phase at ambient pressure. It appears that the nearly spherical and non-polar $\mathrm{CH}_{4}$ molecule can be well represented by single-site models over a wide range of state points. For $\mathrm{NH}_{3}$, the 5-site TraPPE models always underpredicts the experimental high temperature, high pressure densities [25] (and overpredicts the compressibility factor), but falls within $4 \%$ of them. The exp-6 model, fit to these densities, matches even more closely, to within $2 \%$. However, the TraPPE model reproduces the ambient liquid-phase density [26], whereas the exp-6 model underpredicts it by about $10 \%$. The simulations for the TraPPE-UA $\mathrm{CH}_{4}$ and $\mathrm{NH}_{3}$ models were also extended to very 
Table III. Specific densities and sound speeds for $\mathrm{O}_{2}$ and $\mathrm{CH}_{3} \mathrm{OH}$. Subscripts denote the standard deviation in the last digit for simulation data.

\begin{tabular}{|c|c|c|c|c|c|c|c|c|}
\hline \multirow[b]{3}{*}{ Molecule } & \multirow[b]{3}{*}{$\begin{array}{c}T \\
(\mathrm{~K})\end{array}$} & \multirow[b]{3}{*}{$\begin{array}{c}p \\
(\mathrm{MPa})\end{array}$} & \multicolumn{2}{|c|}{ Simulation } & \multirow{2}{*}{\multicolumn{2}{|c|}{$\begin{array}{l}\text { Equation of State } \\
\qquad \exp -6\end{array}$}} & \multirow{2}{*}{\multicolumn{2}{|c|}{ Experiment }} \\
\hline & & & TraPPE-UA & $\exp -6$ & & & & \\
\hline & & & $\begin{array}{c}\rho \\
\left(\mathrm{g} \cdot \mathrm{cm}^{-3}\right)\end{array}$ & $\begin{array}{c}\rho \\
\left(\mathrm{g} \cdot \mathrm{cm}^{-3}\right)\end{array}$ & $\begin{array}{c}\rho \\
\left(\mathrm{g} \cdot \mathrm{cm}^{-3}\right)\end{array}$ & $\begin{array}{c}c \\
\left(\mathrm{~km} \cdot \mathrm{s}^{-2}\right)\end{array}$ & $\begin{array}{c}\rho \\
\left(\mathrm{g} \cdot \mathrm{cm}^{-3}\right)\end{array}$ & $\begin{array}{c}c \\
\left(\mathrm{~km} \cdot \mathrm{s}^{-2}\right)\end{array}$ \\
\hline \multirow[t]{13}{*}{$\mathrm{O}_{2}$} & 80 & 0.101 & $1.138_{2}$ & $1.116_{1}$ & & \multicolumn{3}{|c|}{$1.1906[26]$} \\
\hline & 813 & 2890 & $1.516_{2}$ & $1.60_{1}$ & 1.600 & \multicolumn{3}{|c|}{$3.0_{1}[27]$} \\
\hline & 813 & 2910 & $1.519_{2}$ & $1.61_{1}$ & 1.603 & \multicolumn{3}{|c|}{$3.0_{1}[27]$} \\
\hline & 813 & 3060 & $1.538_{2}$ & $1.63_{1}$ & 1.626 & \multicolumn{3}{|c|}{$3.1_{1}[27]$} \\
\hline & 813 & 3070 & $1.540_{2}$ & $1.63_{1}$ & 1.627 & \multicolumn{3}{|c|}{$3.1_{1}[27]$} \\
\hline & 813 & 3870 & $1.632_{2}$ & $1.73_{2}$ & 1.734 & \multicolumn{3}{|c|}{$3.17_{5}[27]$} \\
\hline & 813 & 7550 & $1.913_{2}$ & $2.06_{4}$ & 2.070 & \multicolumn{3}{|c|}{$3.33_{5}[27]$} \\
\hline & 473 & 510 & $1.061_{2}$ & $1.09_{1}$ & & & & $1.417[24]$ \\
\hline & 473 & 1680 & $1.451_{2}$ & $1.50_{1}$ & & & & $2.357[24]$ \\
\hline & 473 & 3310 & $1.692_{1}$ & $1.77_{2}$ & & & & $3.108[24]$ \\
\hline & 473 & 5610 & $1.890_{1}$ & $2.99_{4}$ & & & & $3.737[24]$ \\
\hline & 473 & 7360 & $1.998_{1}$ & $2.11_{6}$ & & & & $4.089[24]$ \\
\hline & 473 & 10740 & $2.150_{9}$ & $2.30_{7}$ & & & & $4.594[24]$ \\
\hline \multirow[t]{8}{*}{$\mathrm{CH}_{3} \mathrm{OH}$} & 298 & 0.101 & $0.782_{2}$ & $0.8935_{7}$ & & \multicolumn{3}{|c|}{$0.7865[26]$} \\
\hline & 523 & 561 & $0.8845_{3}$ & $1.014_{1}$ & 1.014 & 2.289 & & $2.194[23]$ \\
\hline & 523 & 987 & $0.9700_{3}$ & $1.092_{4}$ & 1.094 & 2.719 & & $2.720[23]$ \\
\hline & 523 & 1424 & $1.0310_{3}$ & $1.149_{6}$ & 1.154 & 3.058 & & $3.000[23]$ \\
\hline & 523 & 1633 & $1.0546_{1}$ & $1.172_{8}$ & 1.178 & 3.197 & & $3.219[23]$ \\
\hline & 523 & 2640 & $1.1431_{1}$ & $1.26_{1}$ & 1.272 & 3.751 & & $3.771[23]$ \\
\hline & 523 & 3160 & $1.1784_{7}$ & $1.29_{1}$ & 1.310 & 3.986 & & $4.023[23]$ \\
\hline & 523 & 3890 & $1.2207_{1}$ & $1.33_{2}$ & 1.357 & 4.278 & & $4.255[23]$ \\
\hline
\end{tabular}

high pressures (5 and $10 \mathrm{GPa}$ ) which most likely fall into the metastable liquid region but nucleation of a solid phase was not observed.

Although there is fairly good agreement between the simulation data using the 3site TraPPE model for $\mathrm{O}_{2}$ and the equation of state data for the exp-6 model (with the TraPPE model yielding densities that are consistently lower by $6 \%$ ), both models predict 


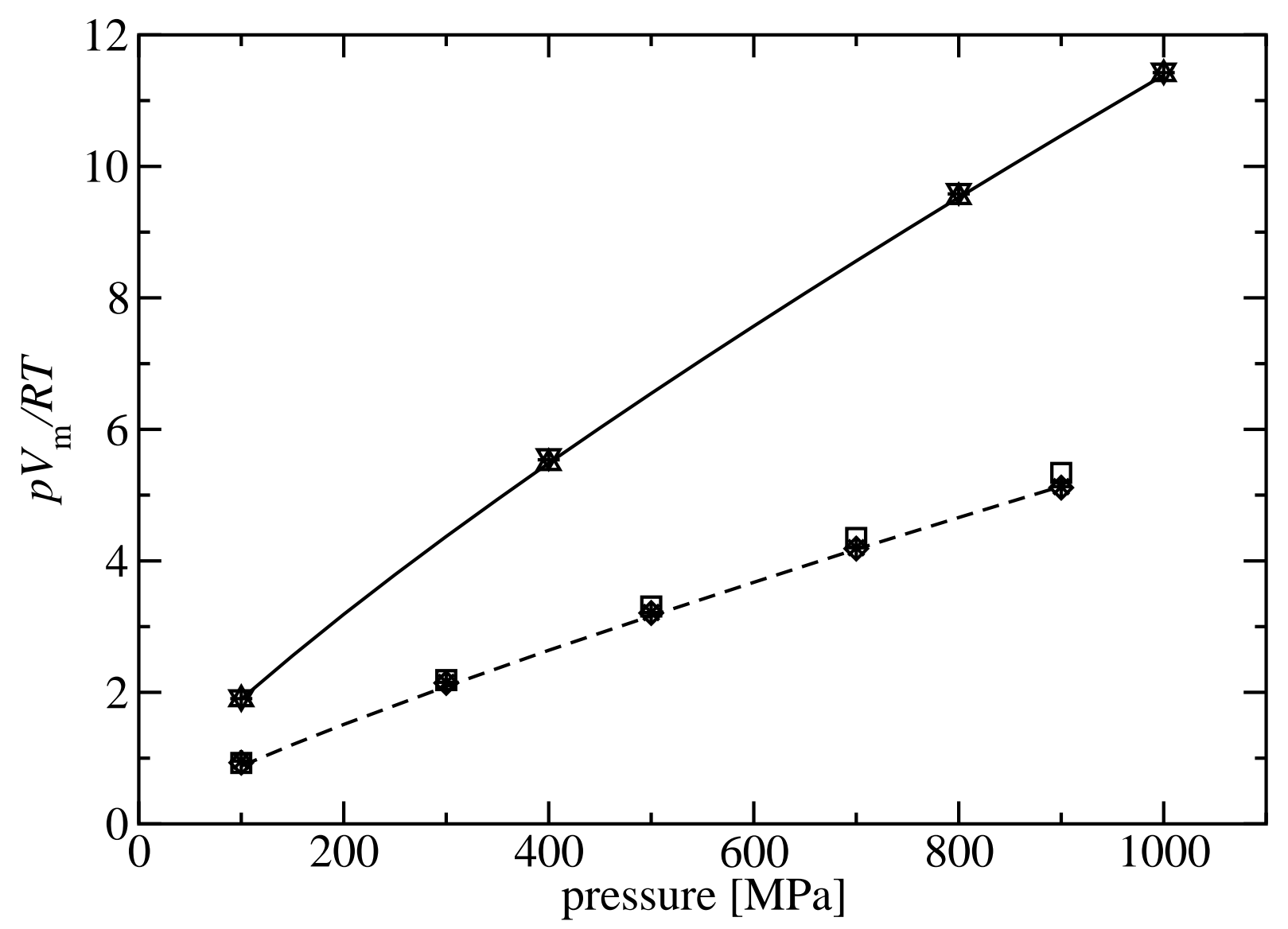

Fig.1. Compressibility factors $\left(Z=P V_{m} / R T\right)$ for $\mathrm{CH}_{4}$ at $298 \mathrm{~K}$ (up triangles represent the TraPPE-UA model, down triangles represent the EOS predictions for the exp-6 model, and crosses represent the simulated exp-6 model) and $\mathrm{NH}_{3}$ at $473 \mathrm{~K}$ (squares represent the TraPPE-UA model, diamonds represent the EOS predictions for the exp-6 model, and stars represent the simulated exp-6 model). Experimental data are depicted as a solid line for $\mathrm{CH}_{4}$ and dashed line for $\mathrm{NH}_{3}$.

densities that fall about a factor of two below the experimental oxygen densities estimated by Johnson et al. [27] from the thermal decomposition of $\mathrm{KClO}_{3}$ into $\mathrm{KCl}$ and $\mathrm{O}_{2}$. Both models somewhat underpredict the liquid-phase density of $\mathrm{O}_{2}$. Given the good agreement between the two models and the fact that the exp-6 model was fitted to sound speed measurements by Abramson et al. [24] and shown to be consistent with shock compression 


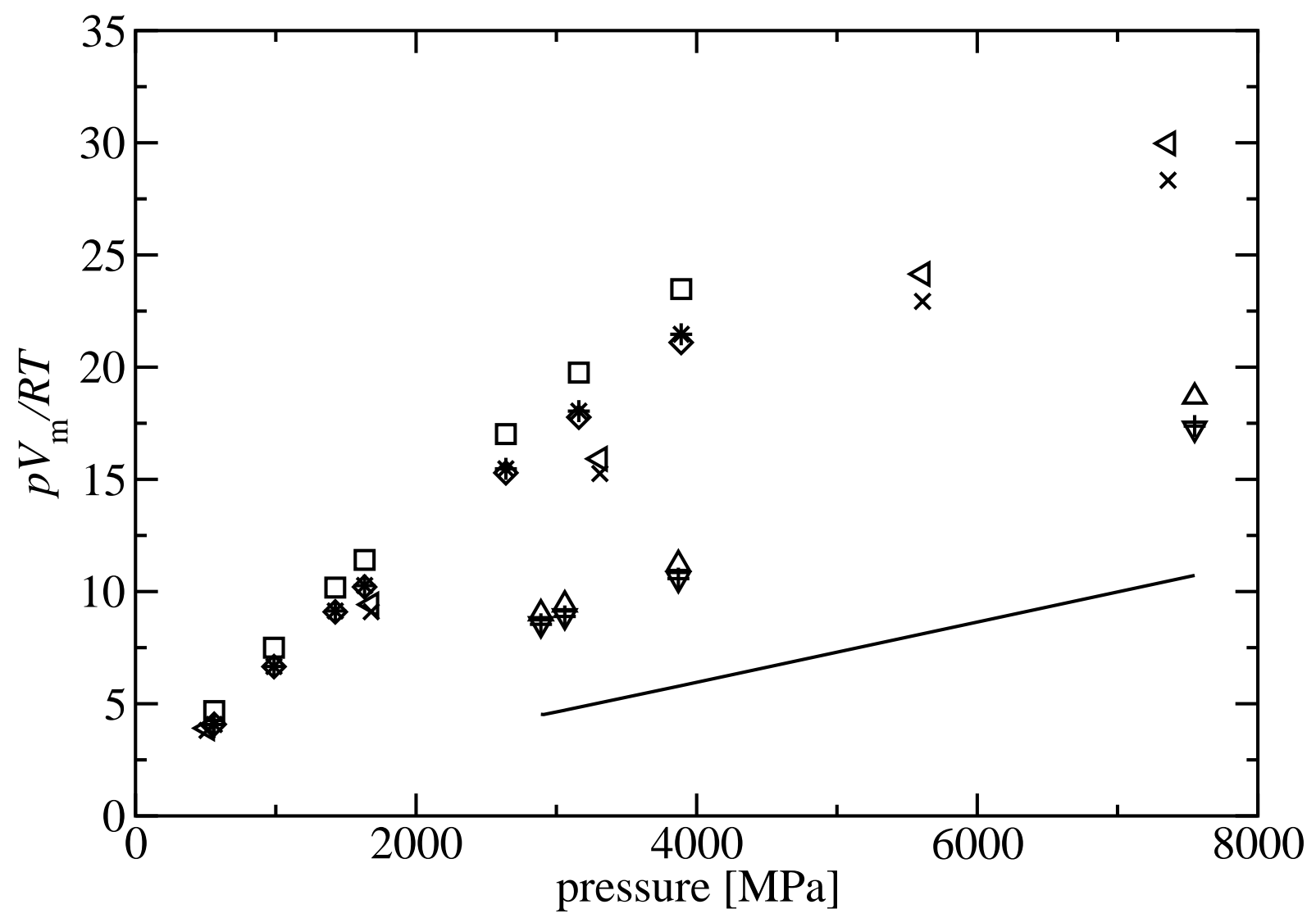

Fig.2. Compressibility factors $\left(Z=P V_{m} / R T\right)$ for $\mathrm{O}_{2}$ at $813 \mathrm{~K}$ (up triangles represent the TraPPE-UA model, down triangles represent the EOS predictions for the exp-6 model, and +'s represent the simulated exp-6 model), $\mathrm{O}_{2}$ at $473 \mathrm{~K}$ (left triangles represent the TraPPE-UA model and $\times$ 's represent the simulated exp-6 model) and $\mathrm{CH}_{3} \mathrm{OH}$ at $523 \mathrm{~K}$ (squares represent the TraPPE-UA model, diamonds represent the EOS predictions for the exp-6 model, and stars represent the simulated exp-6 model). Experimental compressibity factors are available only for $\mathrm{O}_{2}$ at $813 \mathrm{~K}$, and are shown with a solid line.

data [28], it appears that the experimental densities reported by Johnson et al. [27] may not be accurate.

For $\mathrm{CH}_{3} \mathrm{OH}$, we were unable to find experimentally measured densities at high pressures and temperatures well above the critical temperature. Thus, only an indirect com- 
parison can be made to the the experimental sound speeds of Zaug et al. [23] which are well reproduced by the exp-6 model. The densities for the 3 -site TraPPE-UA model fall roughly $12 \%$ below the densities obtained via the equation of state of the exp-6 model. It should be noted that a temperature of $523 \mathrm{~K}$ is rather close to the critical temperature. The $2 \%$ underestimation of the critical temperature by the TraPPE $\mathrm{CH}_{3} \mathrm{OH}$ model yields a reduced temperature of 1.04 at $523 \mathrm{~K}$, whereas the correct value is 1.02 . However, when both models are applied to ambient state points, the TraPPE model yields a liquid-phase density within $1 \%$ of experiment, while the exp-6 model overpredicts it by $14 \%$.

Judging from these four examples, it appears that the TraPPE-UA model has a tendency to somewhat underpredict specific densities at high temperatures and pressures. Part of the discrepancy for the TraPPE densities can be attributed to the use of a LennardJones (LJ) potential which is well known to overestimate the repulsive interactions at high densities considered here (which are sometimes twice as high as the ambient liquid-phase densities used in TraPPE's parametrization).

\section{CONCLUSIONS}

The TraPPE force field fitting philosophy dictates that parameters for a given interaction site should be the same whether that site is in a different molecule or the system is at a different state point. While the fitting is done to vapor-liquid coexistence curves, the TraPPE model can be applied to other high temperature, high pressure state points with only a modest decrease in accuracy. Across all of the state points considered here, the TraPPE models reproduced the experimental densities at extreme conditions with an average error of $6 \%$. The $\exp -6$ models developed specifically for the experimental data studied here matched those values (not surprisingly) much more closely, with an error of about $2 \%$. However, when the same models are applied to ambient conditions, the TraPPE model reproduces experimental data with an average error of $1 \%$, whereas the exp-6 models yield an average error of $9 \%$ at these conditions. When accurate experimental data at 
high pressures and temperatures is available, the fitting methodology of the exp-6 models allows data to be obtained with relative speed and accuracy. When the experimental data is unavailable, or is available for only limited range of state points, simulations using the TraPPE force field can provide reasonably accurate predictions and thereby supplement the sparse experimental data in the high pressure/high temperature regime.

ACKNOWLEDGEMENTS Financial support from the National Science Foundation (CTS-0553911 and ITR-0428774) is gratefully acknowledged. Part of this work was performed under the auspices of the U. S. Department of Energy by the Lawrence Livermore National Laboratory under contract No. W-7405-ENG-48. Part of the computer resources were provided by the Minnesota Supercomputing Institute.

This work was performed under the auspices of the U.S. Department of Energy by University of California, Lawrence Livermore National Laboratory under Contract W-7405-Eng-48. 


\section{REFERENCES}

[1] C. Cavazzoni, G.L. Chiarotti, S. Scandolo, E. Tosatti, M. Bernasconi, and M. Parrinello, Science 283:44 (1999).

[2] H.P. Scott, R.J. Hemley, H.-k. Mao, D.R. Herschbach, L.E. Fried, W.M. Howard, and S. Bastea, Proc. Natl. Acad. Sci. 101:14023 (2004).

[3] M.G. Martin and J.I. Siepmann, J. Phys. Chem. B 102:2569 (1998).

[4] M.G. Martin and J.I. Siepmann, J. Phys. Chem. B 103:4508 (1999).

[5] C.D. Wick, M.G. Martin, and J.I. Siepmann, J. Phys. Chem. B 104:8008 (1999).

[6] B. Chen, J.J. Potoff, and J.I. Siepmann, J. Phys. Chem. B 105:3093 (2001).

[7] J.J. Potoff and J.I. Siepmann, AIChE J. 47: 1676 (2001).

[8] J.M. Stubbs, J.J. Potoff, and J.I. Siepmann, J. Phys. Chem. B 108:17596 (2004).

[9] Z.G. Zhang and Z.H. Duan, J. Chem. Phys. 122:214507 (2005).

[10] C.D. Wick and D.N. Theodorou, Macromolecules 37:7026 (2004).

[11] I.R. McDonald, Mol. Phys. 23:41 (1972).

[12] J.P. Barker and R.O. Watts, Chem. Phys. Lett. 3: 144 (1969).

[13] J.I. Siepmann and D. Frenkel, Mol. Phys. 75:59 (1992).

[14] L. Zhang and J.I. Siepmann, Theor. Chem. Acc. 115:391 (2006).

[15] L. Zhang and J.I. Siepmann, in preparation.

[16] M.P. Allen and D.J. Tildesley, Computer Simulation of Liquids (Oxford University Press, Oxford, 1987). 
[17] M. Ross, J. Chem. Phys. 71:1567 (1979).

[18] H.S. Kang, C.S. Lee, T. Ree, and F.H. Ree, J. Chem. Phys. 82:414 (1985).

[19] L.E. Fried and W.M. Howard, J. Chem. Phys. 109:7338 (1998).

[20] W.B. Brown, J. Chem. Phys. 87:566 (1987).

[21] P.J. Kortbeek and J. A. Schouten, Int. J. Thermophys. 11:455 (1990).

[22] W.J. Nellis, F.H. Ree, M. van Thiel, and A.C. Mitchell, J. Chem. Phys. 75:3055 (1981).

[23] J.M Zaug, L.E. Fried, E.H Abramson, D.W. Hansen, J.C. Crowhurst, and W.M. Howard, High Pressure Research 23:229 (2003).

[24] E.H. Abramson, L.J. Slutsky, M.D. Harrell, and J.M. Brown J. Chem. Phys. 110:10493 (1999).

[25] A. Harlow, G. Wiegand, and E.R. Franck, Ber. Bunsenges. Phys. Chem. 101:1461 (1997).

[26] E.W. Lemmon, M.O. McLinden and D.G. Friend, in NIST Chemistry WebBook, NIST Standard Reference Database Number 69, P.J. Linstrom and W.G. Mallard, eds. (National Institute of Standards and Technology, Gaithersburg MD, 2005), http://webbook.nist.gov.

[27] M.C. Johnson, D. Walker, S.M. Clark, and R.L. Jones, American Mineralogist 86:1367 (2001).

[28] W.J. Nellis and A. C. Mitchell, J. Chem. Phys. 73:6137 (1980). 